Mariana Soares da Silva Peixoto Belo ${ }^{1}$

Wanderlei Pignati

Eliana Freire Gaspar de Carvalho Dores ${ }^{3}$

Josino Costa Moreira ${ }^{4}$

Frederico Peres ${ }^{5}$

\section{Uso de agrotóxicos na produção de soja do Estado do Mato Grosso: um estudo preliminar de riscos ocupacionais e ambientais*}

\author{
Pesticide use in soybean production in Mato Grosso State, Brazil: \\ A preliminary occupational and environmental risk \\ characterization
}

1 Bióloga, doutoranda em Saúde Pública. Escola Nacional de Saúde Pública Sergio Arouca, Fundação Oswaldo Cruz , Rio de Janeiro, RJ, Brasil.

2 Médico, Doutor em Saúde Pública, Professor da Universidade Federal do Mato Grosso, Cuiabá, MT, Brasil.

${ }^{3}$ Bioquímica, Doutora em Química Analítica, Professora da Universidade Federal de Mato Grosso, Cuiabá, MT, Brasil.

${ }^{4}$ Farmacêutico, Doutor em Química Analítica, Pesquisador da Escola Nacional de Saúde Pública Sergio Arouca, Fundação Oswaldo Cruz, Rio de Janeiro, RJ, Brasil.

${ }^{5}$ Biólogo, Doutor em Saúde Coletiva, bolsista da Capes de Estágio Pós-Doutoral no Exterior (Processo BEX 1203-10-0), Pesquisador da Escola Nacional de Saúde Pública Sergio Arouca, Fundação Oswaldo Cruz, Rio de Janeiro, RJ, Brasil.

* O presente manuscrito é parte da tese de doutorado intitulada Percepção de riscos do uso de agrotóxicos em área produtora de soja do estado do $M T$, em elaboração pela aluna Mariana Soares da Silva Peixoto Belo, sob orientação de Frederico Peres. Tese vinculada ao Programa de Pós-graduação em Saúde Pública da Escola Nacional de Saúde Pública Sergio Arouca, Fundação Oswaldo Cruz, Rio de Janeiro, RJ, Brasil.

Projeto subvencionado pelo Edital 18/2006 do CNPq, Processo no 555193/2006-3.

Contato:

Frederico Peres

Centro de Estudos da Saúde do Trabalhador e Ecologia Humana - Escola Nacional de Saúde Pública Sergio Arouca - Fundação Oswaldo Cruz

Rua Leopoldo Bulhões, 1.480 - Sala 29 do CESTEH

CEP 21.041-210

Manguinhos, Rio de Janeiro-RJ

E-mail:

fperes@fiocruz.br

\section{Resumo}

Objetivo: identificar e discutir alguns dos principais riscos associados ao uso de agrotóxicos na produção de soja do estado de Mato Grosso. Método: estudo exploratório descritivo, de caráter preliminar, realizado entre 2008 e 2009 e baseado em triangulação metodológica que incluiu: análise de banco de dados agrícola; análise de indicadores biológicos da exposição a agrotóxicos; e análise da contaminação de água de chuva por esses agentes químicos. Resultados: a análise dos dados de consumo mostra um elevado e crescente uso de agrotóxicos, em particular o do herbicida glifosato. A análise da água de chuva mostrou presença de resíduos de diferentes agrotóxicos, ampliando o risco para além do ambiente de trabalho. Essa exposição ambiental foi detectada pela análise de indicadores biológicos de exposição a agrotóxicos junto a trabalhadores e moradores de áreas próximas às zonas de plantio. Conclusão: os dados do estudo apontam para a necessidade de um monitoramento ambiental e de saúde permanente em áreas produtoras de soja como parte das estratégias de vigilância em saúde do trabalhador e ambiental.

Palavras-chave: agrotóxicos; saúde do trabalhador; saúde ambiental; produção de soja; glifosato.

\begin{abstract}
Objective: To identify and to discuss some of the major risks associated to pesticide use in the soybean production in Mato Grosso State, Midwestern Brazil. Method: It is a descriptive exploratory pilot-study that was carried out between 2008 and 2009, using methodological triangulation, comprising the following: analysis of an agricultural database, analysis of biological indicators of pesticide exposure, and analysis of rainwater contamination by these chemicals. Results: Analysis of pesticides consumption data showed a high and growing use of pesticides in soybean production, particularly glyphosate. Rainwater analysis evidenced the occurrence of different pesticide residues, indicating the amplification of the risks beyond workplace. This environmental exposure was also detected by biological indicator analysis among workers and residents of the plantation neighboring areas. Conclusion: The study data indicate the need for permanent environmental and human health monitoring in soybean production areas as part of workers' health and environmental surveillance strategies.
\end{abstract}

Keywords: pesticides; occupational health; environmental health; soybean production; glyphosate. 


\section{Introdução}

O Brasil é o maior produtor mundial de soja, com uma produção anual de aproximadamente $68 \mathrm{mi}$ lhões de toneladas. A maioria dos estados brasileiros produz soja, com destaque para cinco estados (Mato Grosso, Mato Grosso do Sul, Paraná, Rio Grande do Sul e Goiás) que, juntos, somam $81,55 \%$ de toda a produção nacional (BRASIL, 2010).

Segundo a versão mais recente do Anuário Estatístico do Ministério da Agricultura, Pecuária e Abastecimento (BRASIL, 2005), a produção de soja no país cresceu, entre 1990 e 2005, aproximadamente $260 \%$, passando de uma produção anual de pouco menos de 20 milhões de toneladas para mais de 51 milhões de toneladas em 2005. Houve também, nesse mesmo período de 15 anos, um aumento de mais de $100 \%$ da área colhida, passando de 11,5 mil hectares para quase 23 mil hectares (BRASIL, 2005). Estimativas do Ministério da Agricultura, Pecuária e Abastecimento (BRASIL, 2010) apontam para um crescimento contínuo da produção de soja nos próximos 10 anos a uma taxa de $2,86 \%$ ao ano, o que elevaria a produção nacional a quase 82 milhões de toneladas do grão, ou o equivalente a $40 \%$ do mercado mundial de soja.

O aumento crescente da produção de soja no país tem sido acompanhado pelo aumento também crescente do consumo de herbicidas, particularmente a partir das safras de 2002/2003 e 2003/2004, com a autorização da comercialização - e posteriormente do plantio - de soja geneticamente modificada (MIRANDA et al., 2007; PERES, 2009). Dados sobre a produção de soja no Brasil e o consumo de herbicidas entre 1991 e 2005, compilados por Peres (2009), mostram uma relação positiva entre a produção do grão e o consumo de agrotóxicos dessa classe. Nos Estados Unidos, dados do Departamento de Agricultura (USDA) daquele país (UNITED STATES DEPARTMENT OF AGRICULTURE, 2000 apud PERES, 2009) mostram que essa relação positiva é ainda mais evidente quando se considera a produção de soja transgênica e o consumo do herbicida glifosato, produto destinado, principalmente, ao controle de ervas daninhas nas culturas de soja geneticamente modificada para ser resistente a esse herbicida.

Segundo dados do Sindicato Nacional das Indústrias de Produtos para a Defesa Agrícola - SINDAG (2008), o glifosato é o agrotóxico mais consumido no país, respondendo por quase metade do volume de todos os ingredientes ativos comercializados no Brasil. Tal fato tem uma importância significativa, principalmente se considerarmos que esse herbicida vem sendo apontado, em diversos estudos experimentais e clínicos, como um potencial agente genotóxico (GASNIER et al., 2009; POLETTA et al., 2009), interferente endócrino (GASNIER et al., 2009; HOKANSON et al., 2007) e alergênico (HERAS-MENDAZA et al., 2008; PENAGOS et al., 2004), problemas esses associados à exposição crônica ao glifosato. Assim, apesar da relativa baixa toxicidade aguda, esse agente tem despertado o interesse da comunidade científica (em especial do setor saúde), inclusive no Brasil, onde, desde 2008, o glifosato se encontra sob reavaliação toxicológica pela Agência Nacional de Vigilância Sanitária (2008),

Isso coloca uma situação no horizonte, em que o Brasil, maior consumidor mundial de agrotóxicos na atualidade, tende a ter aumentado seu consumo de herbicidas (principalmente o glifosato), colocando em situação de vulnerabilidade não apenas um grande contingente de trabalhadores rurais, mas também de moradores de áreas próximas aos grandes polos produtores de soja - entre outras grandes monoculturas.

Isto posto, o presente artigo objetiva identificar e discutir alguns dos principais riscos associados ao uso de agrotóxicos na produção de soja do estado de Mato Grosso, evidenciando, principalmente, as dimensões ocupacional e ambiental. É dado destaque a um município da região produtora de soja, Lucas do Rio Verde, localizado a aproximadamente $400 \mathrm{~km}$ ao norte de Cuiabá, capital do estado.

\section{Métodos}

O presente estudo se caracteriza como descritivo-exploratório, de caráter qualitativo, apresentando dados preliminares de um projeto integrado de pesquisa realizado em uma das principais regiões agrícolas do estado do Mato Grosso.

Um grupo de pesquisadores da Fundação Oswaldo Cruz e da Universidade Federal do Mato Grosso elaborou e conduziu, entre o primeiro semestre de 2008 e o primeiro semestre de 2010, um projeto de pesquisa (FUNDAÇÃO OSWALDO CRUZ, 2006) visando a caracterizar os riscos associados à exposição a agrotóxicos (em particular ao glifosato) na produção de soja do município de Lucas do Rio Verde, MT. Os dados aqui apresentados e discutidos foram levantados na primeira etapa desse projeto integrado de pesquisa como subsídio à caracterização de riscos. Esse projeto mais abrangente era composto pelas seguintes etapas: a) análise de resíduos de agrotóxicos em amostras ambientais (água e sedimento de rios e córregos, ar e água de chuva); b) análise de indicadores biológicos junto a trabalhadores e moradores da região; c) levantamento de dados epidemiológicos em bases 
de dados estaduais e municipais; d) monitoramento ecotoxicológico de espécies bioindicadoras; e e) análise da percepção de risco de moradores e trabalhadores do município.

Para subsidiar as etapas supramencionadas, foi planejado e realizado, entre 2008 e 2009, um estudo preliminar visando a identificar os perigos associados à produção de soja no estado do Mato Grosso. Este artigo apresenta e discute os resultados desse estudo preliminar, realizado em duas etapas distintas. A primeira, ocorrida no ano de 2008, compreendeu: a) o levantamento do consumo de agrotóxicos no estado junto ao Instituto de Defesa Agropecuária do Mato Grosso (2008); b) e a observação participante em dois municípios do estado reconhecidos como grandes produtores de soja. Para a análise dos dados referentes ao item "a", uma vez de posse dos bancos de dados fornecidos pelo órgão mencionado, foram organizadas planilhas em Excel (Microsoft Corporation) e utilizaram as ferramentas estatísticas desse software para o tratamento dos dados. Os dados de observação participante (item "b") foram registrados em caderneta de campo.

Entre dezembro de 2008 e junho de 2009, foi realizada a segunda etapa do estudo preliminar, que incluiu: a) análise de indicadores biológicos de exposição a agrotóxicos junto a trabalhadores e residentes do município de Lucas do Rio Verde; e b) análise da contaminação da água de chuva por agrotóxicos nesse mesmo município.

Cabe ressaltar que essa segunda etapa do estudo não se caracteriza como uma avaliação da exposição. Utilizaram-se apenas indicadores biológicos de exposição - no caso, indicadores de dose interna: presença de resíduos de agrotóxicos e/ou seus metabólitos (GIL; PIA, 2001; KLAASSEN; AMDUR; DOULL, 2001) - e presença de resíduos de agrotóxicos na água de chuva como forma de identificar, respectivamente, a possível exposição de indivíduos a agrotóxicos e o possível acúmulo de resíduos desses agentes químicos na água de chuva da região.

Para as análises de indicadores biológicos de exposição (urina e sangue), foram selecionados 79 indivíduos de Lucas do Rio Verde, sendo 42 trabalhadores rurais da comunidade de São Cristóvão (uma das localidades mais produtivas do município) e 37 moradores do centro da cidade (zona urbana do município). Nessas amostras biológicas, foram analisados indicadores de dose interna através de dois métodos: a) nas amostras de sangue, dosou-se a presença de 27 diferentes resíduos de inseticidas organoclorados, utilizando-se técnicas cromatográficas (SARCINELLI, 2001); b) nas amostras de urina, dosou-se a presença de resíduos de inseticidas piretroides e do herbicida glifosato utilizando-se kits colorimétricos com leitura em ELISA (BERNAL; SOLOMON; CARRASQUILLA, 2009; WATANABE et al., 2005) .

A amostragem - de caráter qualitativo (não significativa estatisticamente) e do tipo bola de neve, na qual um indivíduo, ao ser abordado e aceitar participar do estudo, indica o próximo indivíduo a ser consultado (BECKER, 2004) - foi determinada em função daqueles trabalhadores e moradores que se dispuseram a participar de todas as etapas do estudo, incluindo um estudo de percepção de risco, realizado posteriormente (2009 e 2010) em conformidade com os critérios estabelecidos no projeto de pesquisa (FUNDAÇÃO OSWALDO CRUZ, 2006).

A metodologia para a análise da contaminação da água de chuva foi adaptada e validada na dissertação de mestrado realizada por Santos (2010), que foi bolsista deste projeto e analisou os resíduos de agrotóxicos. Para a realização do trabalho, optou-se pela instalação de coletores pluviométricos em quatro pontos do município de Lucas do Rio Verde. As amostras foram coletadas quinzenalmente (1 frasco coletado a cada 15 dias, completando 39 amostras em cada uma das quatro localidades e totalizando 156 amostras coletadas durante a safra de 2008/2009), mantidas refrigeradas e, a cada dois meses, analisadas pelo método de extração em fase sólida C-18 (identificação e quantificação em cromatógrafo a gás acoplado a espectrômetro de massa - CG/ EM). Essa adaptação permitiu a análise de resíduos de atrazina, clorpirifós, endosulfan (alfa e beta), flutriafol, malationa, metalacloro e metil paration.

Os coletores para a amostragem de água de chuva foram instalados em dois locais de zona rural e dois em área urbana. Na zona rural, os coletores foram instalados nas localidades de São Cristóvão e Itambiquara (nos limites sul e norte, respectivamente, do município). A comunidade rural de São Cristóvão possui 98 famílias, dista $35 \mathrm{~km}$ ao sul da sede do município e é uma das localidades mais produtivas da região. As coletas foram realizadas no pátio da escola, local onde foi montado um coletor acoplado a recipiente de vidro âmbar. Na localidade de Itambiquara, que possui 52 famílias e dista $30 \mathrm{~km}$ da zona urbana/sede do município, o coletor foi colocado próximo à residência de um produtor de soja. Em ambas as localidades, a produção de soja é intensa e realizada a partir de processos de trabalho bastante similares (alta mecanização, utilização de vasto território para plantio, pulverização aérea ou com trator-dispersor de alta pressão e elevado consumo de agrotóxicos, principalmente herbicidas e fungicidas). Na zona urbana, os coletores foram colocados no Colégio Dom Bosco (situado no alto de um morro/elevação, na parte central da área urbana) e no Centro Tecnológico (Cetec), localizado 
em bairro periférico da cidade (mas ainda pertencente à zona urbana).

Os dados obtidos, analisados e aqui apresentados são considerados preliminares, visto que o estudo teve continuidade até janeiro de 2010 e que novas análises foram realizadas, tanto no município de Lucas do Rio Verde, quanto em outro município do estado, Campo Verde. Por esta razão também classificamos o presente estudo como exploratório e de caráter preliminar.

Todas as etapas da pesquisa respeitaram a Resolução 196/96 do Conselho Nacional de Saúde, tendo o projeto sido submetido e aprovado pelo Comitê de Ética em Pesquisa da Escola Nacional de Saúde Pública.

\section{Resultados}

O estado do Mato Grosso é o maior produtor nacional de soja, com uma produção aproximada de 19 milhões de toneladas na safra 2009/2010, ou o equivalente a aproximadamente $28 \%$ da produção brasileira (BRASIL, 2010). Entre 1990 e 2005, esse estado foi o que registrou maior aumento entre todos os estados produtores de soja no país, com sua produção anual passando de pouco mais de três milhões de toneladas em 1990 para 17,7 milhões, um aumento de quase 500\% (BRASIL, 2005).

A partir de janeiro de 2005, o Instituto de Defesa Agropecuária do Estado do Mato Grosso (INSTITUTO DE DEFESA AGROPECUÁRIA, 2008) instituiu no estado um sistema de informação de agrotóxicos organizado através das informações contidas nas notas fiscais e de dados retirados dos receituários agronômicos emitidos no MT.
A análise desse banco de dados, atualizado até 2007 (INSTITUTO DE DEFESA AGROPECUÁRIA, 2008), aponta para uma associação entre a produção de soja no estado e o elevado consumo de agrotóxicos. No município de Lucas do Rio Verde, um dos maiores produtores de soja do estado, observa-se tal relação (quando comparado a outros municípios do estado com intensa atividade agrícola, mas não produtores de soja), caracterizada pelo elevado consumo de algumas classes de agrotóxicos, como os herbicidas e os fungicidas (aquelas mais frequentemente utilizadas na cultura da soja), em relação às demais.

No caso dos inseticidas, seu consumo está associado tanto à cultura da soja, quanto ao cultivo do milho, em particular aquele realizado imediatamente após a colheita da soja, também chamado de "safrinha do milho" (INSTITUTO DE DEFESA AGROPECUÁRIA, 2008).

Na Tabela 1 é possível observar o aumento significativo (aproximadamente 191\%), em apenas três anos, do consumo de agrotóxicos de Classe Toxicológica IV - Pouco tóxicos (AGÊNCIA NACIONAL DE VIGILÂNCIA SANITÁRIA, 2011). Nesta classe, incluem-se algumas formulações do herbicida glifosato, inclusive o Round Up, produto formulado mais utilizado em Lucas do Rio Verde, e alguns fungicidas utilizados na cultura de soja do município - em particular os dos grupos triazol e estrobilurina ou suas associações (INSTITUTO DE DEFESA AGROPECUÁRIA, 2008). Tal dado é corroborado pelas informações contidas na Tabela 2, que mostram o aumento do consumo dessas classes de agrotóxicos entre os anos de 2005 e 2007. Apesar do aumento significativo na classe IV, não houve uma diminuição expressiva nas classes de maior toxicidade, o que compõe para uma situação de especial atenção quanto aos riscos associados ao elevado consumo dos agrotóxicos observados.

Tabela 1 Consumo de agrotóxicos por classe toxicológica (em litros de produtos formulados) em Lucas do Rio Verde, MT, 2005 a 2007

\begin{tabular}{lcccc}
\hline \multicolumn{1}{c}{ Classe toxicológica } & 2005 & 2006 & 2007 & Total geral \\
\hline I - Extremamente tóxico & 1.487 .903 & 1.221 .600 & 1.037 .454 & 3.746 .957 \\
II - Altamente tóxico & $368.411,7$ & $329.066,5$ & 352.672 & 1.050 .150 \\
III - Medianamente tóxico & 1.232 .094 & 1.203 .346 & 1.058 .054 & 3.493 .494 \\
IV - Pouco tóxico & $566.106,3$ & 1.435 .034 & 1.649 .019 & 3.650 .160 \\
Muito pouco tóxico & 1.968 & 1.556 & 1.101 & 4.625 \\
\hline Total geral & 3.656 .483 & 4.190 .603 & 4.098 .300 & 11.945 .387 \\
\hline
\end{tabular}

Fonte: Instituto de Defesa Agropecuária do Estado do Mato Grosso (2008) 
Tabela 2 Consumo de agrotóxicos por classe de uso do produto (em litros de produtos formulados) em Lucas do Rio Verde, MT, 2005 a 2007

\begin{tabular}{lcccc}
\hline \multicolumn{1}{c}{ Classe de uso do produto } & 2005 & 2006 & 2007 & Total geral \\
\hline Adjuvante & 21.306 & 155.557 & 91.635 & 268.498 \\
Espalhante adesivo & 23.967 & 2.818 & 6.987 & 33.772 \\
Fungicida & 176.664 & 467.157 & 347.012 & 990.833 \\
Herbicida & 2.039 .276 & 2.292 .102 & 2.479 .676 & 6.811 .054 \\
Inseticida & 1.303 .414 & 1.195 .816 & $995.766,4$ & 3.494 .996 \\
Inseticida e acaricida & 55.185 & 30.794 & $108.918,3$ & $194.897,3$ \\
Inseticida e adjuvante & 4.140 & 29.279 & 17.900 & 51.319 \\
Regulador de crescimento & 32.532 & 17.080 & 50.406 & 100.018 \\
\hline Total geral & 3.656 .483 & 4.190 .603 & 4.098 .300 & 11.945 .387 \\
\hline
\end{tabular}

Fonte: Instituto de Defesa Agropecuária do Estado do Mato Grosso (2008)

Os dados apresentados na Tabela 3 apontam para um consumo intenso de agrotóxicos em extensa área do município (crescente desde o final da década de 1990) dedicada a lavouras temporárias (principalmente soja e milho). Considerando dados coletados na etapa de observação participante, que mostram que as plantações ocupam a maior parte do município e que a distância entre as residências e as áreas de plantio é mínima, inferior a 20 m (FUNDAÇÃO OSWALDO CRUZ, 2006), é possível antecipar situações de exposição a agrotóxicos nesse município, principal justificativa para a realização de análise de indicadores biológicos de exposição a agrotóxicos.

Quanto à análise desses indicadores biológicos, nas amostras de urina foi detectada a presença de resíduos de glifosato e piretroides. Para o glifosato, foram observados $88 \%$ de amostras positivas e, para os piretroides, $80 \%$ de amostras positivas. Nas amostras de sangue, foi observado que $61 \%$ dessas amostras foram positivas para, pelo menos, um determinado inseticida organoclorado. Os resultados dessas análises encontram-se apresentados na Tabela 4.

Apesar da adoção de amostra qualitativa (não representativa estatisticamente e com "n" amostral pequeno), o que não nos permite fazer extrapolações confiáveis, foi possível observar que os níveis de glifosato em alguns indivíduos trabalhadores e residentes na zona rural era superior a dos doadores da zona urbana. Tal fato pode estar relacionado à manipulação desses compostos no processo de produção da soja (principalmente) e, em menor escala, do milho. Quanto aos piretroides, observam-se níveis mais elevados entre alguns moradores da área urbana, o que pode ser explicado, entre diversos fatores, pela possível influência dos agrotóxicos utilizados no controle de vetores no domicílio e nos bairros/ruas da zona urbana (principalmente no combate à dengue). Esses dados, entretanto, apontam para a possibilidade de existência de exposições distintas entre moradores das zonas urbana e rural, dado que deve ser confirmado e aprofundado em estudos posteriores.

Com relação à contaminação da água de chuva por agrotóxicos (Tabela 5), foi possível observar a presença de resíduos de diferentes agrotóxicos no sistema pluviométrico, representando uma pouco estudada via de contaminação que transcende o ambiente de trabalho e amplifica o risco da exposição a esses agentes químicos.

Um fator limitante dessa amostragem foi a impossibilidade de se dosar, pelo mesmo método (cromatográfico), os resíduos de glifosato, o agrotóxico mais utilizado na região. (INSTITUTO DE DEFESA AGROPECUÁRIA, 2008) Isso devido à dificuldade de se seguir os protocolos de análise internacionalmente validados e à ausência, na região, de equipamentos analíticos disponíveis para esse tipo de análise.

Além dos limites anteriormente identificados (amostragem qualitativa, pequena amostra de indivíduos participantes das análises de indicadores biológicos e impossibilidade de dosar o glifosato em água de chuva), outros fatores se apresentam como limitadores da abrangência dos resultados desse estudo preliminar, exploratório, incluindo: a) impossibilidade de se contar com dados epidemiológicos que pudessem corroborar com os indicativos levantados; b) a necessidade de se produzir dados preliminares em espaço de tempo relativamente curto (pouco mais de um ano) para subsidiar o projeto de pesquisa mais abrangente (FUNDAÇÃO OSWALDO CRUZ, 2006); c) e a dificuldade de se padronizar metodologias para analisar os mesmos contaminantes em diferentes compartimentos (biológicos e ambientais). 
Tabela 3 Matriz de produção agrícola de Lucas do Rio Verde, MT, para lavouras temporárias (1998 a 2007)

\begin{tabular}{|c|c|c|c|c|c|c|c|c|c|c|}
\hline & 1998 & 1999 & 2000 & 2001 & 2002 & 2003 & 2004 & 2005 & 2006 & 2007 \\
\hline \multicolumn{11}{|l|}{ Produção agrícola } \\
\hline $\begin{array}{l}\text { Lavoura temporária } \\
\text { (1.000 hectares) }\end{array}$ & 197 & 251 & 249 & 266 & 311 & 347 & 334 & 380 & 373 & 412 \\
\hline $\begin{array}{l}\text { Produção lavoura temporária } \\
\text { (1.000 toneladas) }\end{array}$ & 462 & 676 & 805 & 785 & 969 & 1.310 & 916 & 1.314 & 1.317 & 1.405 \\
\hline \multicolumn{11}{|l|}{ Insumos agrícolas } \\
\hline Agrotóxicos (1.000 litros) & 1.899 & 2.413 & 2.397 & 2.563 & 2.992 & 3.340 & 3.208 & 3.656 & 4.191 & 4.098 \\
\hline Agrotóxicos (litro/hectare) & 10 & 10 & 10 & 10 & 10 & 10 & 10 & 10 & 11 & 10 \\
\hline Agrotóxicos (litro/habitante) & 130,33 & 156,11 & 124,11 & 122,82 & 136,19 & 143,92 & 131,31 & 134,33 & 146,29 & 136,35 \\
\hline
\end{tabular}

Fonte: Instituto de Defesa Agropecuária do Estado do Mato Grosso (2008)

Tabela 4 Análises de resíduos de agrotóxicos em amostras biológicas de residentes da área urbana e trabalhadores/residentes da zona rural do município de Lucas do Rio Verde, MT (março de 2009)

\begin{tabular}{|c|c|c|c|c|c|}
\hline \multirow{2}{*}{ Tipo de amostra } & \multirow{2}{*}{ Agrotóxicos } & \multicolumn{2}{|c|}{ Amostras positivas } & \multicolumn{2}{|c|}{$\begin{array}{c}\text { Concentração } \\
\text { (valores mínimos e máximos) }\end{array}$} \\
\hline & & $\begin{array}{l}\text { Urbana } \\
(n=37)\end{array}$ & $\begin{array}{c}\text { Rural } \\
(n=42)\end{array}$ & Urbana & Rural \\
\hline \multirow{2}{*}{ Urina } & Glifosato & 35 & 35 & $0,21-3,35 \mathrm{ppb}$ & 0,38-5,05 ppb \\
\hline & Piretroides & 33 & 30 & $0,41-22,31 \mathrm{ng} / \mathrm{ml}$ & $0,46-13,26 \mathrm{ng} / \mathrm{ml}$ \\
\hline \multirow{4}{*}{ Sangue total } & Aldrin & - & 4 & - & $0,7-4,41 \mathrm{ng} / \mathrm{ml}$ \\
\hline & $\mathrm{p}, \mathrm{p}$ 'DDE & 18 & 24 & $0,40-16,91 \mathrm{ng} / \mathrm{ml}$ & $0,16-14,65 \mathrm{ng} / \mathrm{ml}$ \\
\hline & o,p'DDT & - & 1 & - & $0,40 \mathrm{ng} / \mathrm{ml}$ \\
\hline & p,p'DDT & - & 5 & - & $0,48-1,65 \mathrm{ng} / \mathrm{ml}$ \\
\hline
\end{tabular}

Fonte: Elaboração própria.

Tabela 5 Resíduos de agrotóxicos em amostras de água de chuva coletadas em quatro pontos de Lucas do Rio Verde, MT, por faixa de concentração ( $\mu \mathrm{g} / \mathrm{L})$ e frequência de detecção (\%), entre dez/2008 a jun/2009

\begin{tabular}{lcccc}
\hline \multicolumn{1}{c}{ Agrotóxicos } & $\begin{array}{c}\text { Itambiquara } \\
\text { Rural } \\
(n=39)\end{array}$ & $\begin{array}{c}\text { Dom Bosco } \\
\text { Urbano } \\
(n=39)\end{array}$ & $\begin{array}{c}\text { São Cristóvão } \\
\text { Rural } \\
(n=39)\end{array}$ & $\begin{array}{c}\text { Periferia (Cetec) } \\
\text { Urbano } \\
(n=39)\end{array}$ \\
\hline $\begin{array}{l}\text { Atrazina } \\
\text { Clorpirifós }\end{array}$ & $0,01-3,82 \mu \mathrm{g} / \mathrm{L}(35 \%)$ & $0,01-1,87 \mu \mathrm{g} / \mathrm{L}(44 \%)$ & $0,01-1,11 \mu \mathrm{g} / \mathrm{L}(25 \%)$ & $0,01-0,27 \mu \mathrm{g} / \mathrm{L}(40 \%)$ \\
$\alpha$ - Endossulfan & $\mathrm{Nd}$ & $\mathrm{Nd}$ & $\mathrm{Nd}$ & $\mathrm{Nd}$ \\
$\beta$ - Endosulfan & $0,01-0,39 \mu \mathrm{g} / \mathrm{L}(35 \%)$ & $0,01-0,52 \mu \mathrm{g} / \mathrm{L}(17 \%)$ & $0,01-0,4 \mu \mathrm{g} / \mathrm{L}(37 \%)$ & $\mathrm{Nd}$ \\
Flutriafol & $\mathrm{Nd}$ & $0,01-0,44 \mu \mathrm{g} / \mathrm{L}(39 \%)$ & $\mathrm{Nd}$ & $\mathrm{Nd}$ \\
Malationa & $0,01-0,06 \mu \mathrm{g} / \mathrm{L}(25 \%)$ & $0,01-0,05 \mu \mathrm{g} / \mathrm{L}(22 \%)$ & $\mathrm{Nd}$ & $\mathrm{Nd}$ \\
Metalacloro & $0,01-0,16 \mu \mathrm{g} / \mathrm{L}(50 \%)$ & $0,01-0,44 \mu \mathrm{g} / \mathrm{L}(33 \%)$ & $\mathrm{Nd}$ & $0,01-0,32 \mu \mathrm{g} / \mathrm{L}(20 \%)$ \\
Metil paration & $0,01-0,12 \mu \mathrm{g} / \mathrm{L}(25 \%)$ & $0,01-0,20 \mu \mathrm{g} / \mathrm{L}(17 \%)$ & $\mathrm{Nd}$ & $\mathrm{Nd}$ \\
\hline
\end{tabular}

$\mathrm{Nd}=$ não detectado

Fonte: Santos (2010) 


\section{Discussão}

A produção de soja no estado do Mato Grosso aparece como fator gerador de riscos à saúde do trabalhador rural e dos habitantes do entorno de áreas produtivas, principalmente no que diz respeito à exposição a agrotóxicos. As características do cultivo de soja, que incluem o uso extensivo de terras, o alto índice de mecanização e o intensivo uso de agrotóxicos, acabam por determinar um panorama de exposição ambiental amplificado, no qual os riscos relacionados com o uso desses agentes químicos são extrapolados para além da plantação. Isso coloca não apenas os trabalhadores rurais envolvidos com esse cultivo em situação de risco, mas sua família e os habitantes de áreas próximas (ou nem tão próximas assim, como veremos) às zonas de plantio. Os resultados apresentados no presente estudo apontam para alguns dos impactos do uso de agrotóxicos na produção de soja sobre a saúde humana e o ambiente.

Entre os diferentes agrotóxicos usados na produção de soja no estado, é necessário destacar o glifosato, ou seu produto formulado mais comum, o Round Up (INSTITUTO DE DEFESA AGROPECUÁRIA, 2008), o agente mais frequentemente associado ao cultivo de soja, principalmente a geneticamente modificada, e que, nos últimos anos, vem levantando suspeitas na comunidade científica pelo seu potencial de alteração (disrupção) do sistema endócrino humano (CURWIN et al., 2002; FARIA; ROSA; FACCHINI, 2009; SOLOMON; MARSHALL; CARRASQUILLA, 2009; VARONA et al., 2009), assim como por seu potencial genotóxico (GASNIER et al., 2009; POLETTA et al., 2009; MLADINIC et al., 2009), alérgico (HERAS-MENDAZA et al., 2008; PENAGOS et al., 2004; NIELSEN; NIELSEN; SORENSEN, 2007; SLAGER et al., 2010) e de problemas no fígado (CHALUBINSKI; KOWALSKI, 2006; EJAZ et al., 2004).

Existe, hoje, uma crescente literatura acerca do tema que se baseia tanto em estudos in vitro (GASNIER et al., 2009; HOKANSON et al., 2007), em dados epidemiológicos (CURWIN et al., 2002; SOLOMON; MARSHALL; CARRASQUILLA, 2009), como em estudos utilizando outras espécies de animais (BRAKE; EVERSON, 2004; DALLEGRAVE et al., 2003).

Seja qual for o desenho do estudo ou efeito à saúde observado na literatura internacional, ficam as evidências do potencial nocivo desses agentes químicos sobre o organismo humano (em especial sobre o sistema endócrino), problemas que, em virtude do tipo de exposição e da baixa toxicidade aguda desses agentes químicos, podem ser percebidos apenas após alguns anos e, quando percebidos os sintomas, os danos já podem ser irreversíveis (CURWIN et al., 2002; SOLOMON; MARSHALL; CARRASQUILLA, 2009; VARONA et al., 2009).
Ademais, a inexistência de limites de segurança para exposição ao herbicida glifosato e contaminações por ele, assim como para os inseticidas das classes dos organoclorados e dos piretroides, também coloca incertezas quanto ao desenho de um programa de monitoramento (por meio de indicadores biológicos) próprio para trabalhadores e não trabalhadores potencialmente expostos a esses agentes químicos. Isto coloca a necessidade de um olhar mais cuidadoso sobre a exposição a estes agentes químicos e as decorrências para a saúde dos trabalhadores rurais, constituindo um desafio para a vigilância em saúde.

\section{Quando os riscos ultrapassam o ambiente de trabalho}

Os dados apresentados na Tabela 5 apontam para uma deriva ambiental de resíduos de agrotóxicos (provavelmente aqueles utilizados nas atividades agrícolas) e sua concentração na água da chuva. A análise das amostras também aponta para uma situação na qual tanto trabalhadores, quanto moradores (sejam da zona rural ou urbana) estão expostos a diferentes tipos de agrotóxicos através de rota ambiental (nesse caso específico, a água das chuvas).

Diversos fatores relacionados contribuem para que a exposição aos agrotóxicos utilizados na produção de soja do estado do Mato Grosso ultrapasse os limites do ambiente de trabalho. Dentre esses fatores, destacamos:

- o alto nível de mecanização associado a essa cultura e a necessidade de uso de equipamentos dispersores de grande vazão;

- as grandes dimensões de terra utilizadas para a plantação da soja, que levam ao uso de aviões ou tratores pulverizadores, aumentando o raio de dispersão (deriva) desses resíduos de agrotóxicos; e

- as altas temperaturas observadas na região, aumentando a volatilização e a dispersão desses agentes químicos, tornando-os passíveis de serem transportados pelos fortes ventos da região e/ou se concentrarem em forma de vapores nas nuvens.

Com relação aos dados obtidos através da análise da contaminação de água de chuva por agrotóxicos, como não há, na legislação nacional, previsão de limites de segurança para resíduo de agrotóxicos em água de chuva, fica difícil precisar a extensão e o risco a que esses indivíduos, trabalhadores e população em geral, estão expostos.

Outros autores também encontraram resultados semelhantes aos aqui apresentados, na França (SCHEYER et al., 2006; SCHUMMER et al., 2009), na Grécia (ROUVALIS et al., 2009) e na Bélgica (QUAGHEBEUR et al., 2004), países onde também não há limites de confiança/segurança para resíduos 
de agrotóxicos em água de chuva. Nesses estudos, é possível observar alguns pontos de concordância no que diz respeito às recomendações apresentadas pelos autores e aquelas identificadas após a análise dos dados do estudo-piloto aqui apresentados:

- na ausência de limites de tolerância confiáveis para resíduos de agrotóxicos em água de chuva, o simples registro desses resíduos deve ser observado como indicativo para ações de vigilância em saúde que incluam os tomadores de decisão envolvidos com o problema, como gestores dos setores da saúde, do meio ambiente e da agricultura, educadores, profissionais de saúde e grupos organizados de trabalhadores e moradores de áreas próximas à plantações;

- testes visando a caracterizar o impacto dessa água de chuva sobre algumas espécies-alvo (bioindicadores) devem ser realizados quando detectados resíduos de agrotóxicos em água de chuva, aumentando o espectro e a confiabilidade dos dados disponíveis para o processo de gerenciamento de riscos;

- associados às análises ambientais, outros testes, tais como análises clínicas e toxicológicas, devem ser empregados, visando a caracterizar com mais profundidade a situação de vulnerabilidade em que se encontram tanto trabalhadores e população vizinha a áreas de intenso uso de agrotóxicos, como as regiões produtoras de soja.

Não desconsiderando a importância da produção agrícola no país - em particular da cadeia produtiva da soja no estado do Mato Grosso -, faz-se necessário encontrar caminhos que garantam a primazia da saúde face ao desenvolvimento econômico. Enquanto as estratégias de vigilância em saúde forem negligenciadas ou colocadas em segundo plano, teremos que continuar lidando com e tratando de problemas e doenças evitáveis que, ano após ano, traduzem-se em custos para o setor da saúde e contribuem para a deterioração da qualidade de vida e de trabalho de nossa população.

Este modelo perigoso, particularmente evidenciado nos países em desenvolvimento onde as cadeias agropecuárias ainda têm papel dominante na economia, leva à necessidade da garantia da sustentabilidade dos processos produtivos, que se inicia, em primeiro lugar, com o cuidado e a promoção da saúde entre os trabalhadores e as populações residentes em áreas de intensa produção.

A experiência de alguns projetos e estudos realizados em países como os EUA (ARCURY et al., 2009; SHIPP et al., 2007) e a África do Sul (LONDON; BAILIE, 2001), além de estudos prospectivos/ de modelagem realizados no Brasil (SOARES; MORO;
ALMEIDA, 2002), tem demonstrado que a prevenção dos riscos à saúde causados por agrotóxicos pode ser compensadora do ponto de vista econômico (ou seja, os gastos com a perda da produção agrícola causada pela diminuição do uso de agrotóxicos é menor que os gastos com a saúde da população exposta a esses agentes químicos), em especial quando consideramos alguns problemas de saúde (como aqueles potencialmente associados ao glifosato) em que os sintomas não são imediatamente visíveis e, quando o são, muito pouco se tem a fazer em termos da atenção à saúde. Nesse sentido, uma estratégia integrada de análise e gerenciamento de riscos pode contribuir para a melhoria da qualidade de vida de milhares de indivíduos que hoje se encontram em situação de extrema vulnerabilidade, determinada pelo uso intensivo de agrotóxicos no país.

\section{Considerações finais}

Apesar das limitações, os dados apresentados e discutidos no presente artigo desvelam um problema de grande importância para a saúde pública nacional, com trabalhadores e populações vizinhas a áreas de produção de soja em situação de vulnerabilidade face aos efeitos nocivos dos agrotóxicos sobre a saúde humana. A compreensão da dimensão e da importância desse problema só é possível através da análise dos riscos ocupacionais e ambientais correlatos às diferentes etapas da produção da soja, não apenas no estado de Mato Grosso (ou no polo produtor de soja do estado), mas em todas as regiões produtoras do país, que na atualidade ocupa o primeiro lugar no ranking de produção dessa commodity agrícola.

A análise de indicadores biológicos entre trabalhadores rurais e indivíduos residentes na área urbana do município de Lucas do Rio Verde apontou para a possibilidade de diferenciação da exposição a agrotóxicos. Indivíduos trabalhadores e residentes nas áreas de produção agrícola (zona rural) podem estar mais expostos ao glifosato, usado na produção da soja, ao mesmo tempo em que os moradores da zona urbana podem se encontrar mais expostos aos piretroides, utilizados no combate a vetores de doenças. Essa diferenciação precisa ser melhor evidenciada, razão pela qual se faz necessária a realização de estudos posteriores para confirmar e aprofundar os resultados aqui obtidos.

A ocorrência de resíduos de agrotóxicos na água de chuva amplifica significativamente o espectro da exposição ambiental a que a maioria dos residentes em áreas de produção de soja (não importando o local de residência, em zona rural ou urbana) está sujeita. Esses indicativos devem estar integrados a estratégias de avaliação e gerenciamento de riscos que incluam não apenas o monitoramento ambiental, mas também ações de vigilância em saúde/saúde do 
trabalhador. Ademais, é necessário incluir a avaliação do impacto desses resíduos de agrotóxicos sobre espécies não alvo (bioindicadores), uma vez que a precipitação desses resíduos ocasiona sua deposição em diversos compartimentos biológicos, principalmente em cursos d'água, aumentando o espectro do risco e colocando uma nova rota de exposição/contaminação (via consumo de água contaminada pela chuva e/ou de peixes coletados em locais onde há essa deposição de água de chuva).
Os resultados do estudo apontam para a importância das estratégias de avaliação e gerenciamento de riscos como ações de vigilância em saúde. Sabe-se que a análise e o gerenciamento de riscos de problemas determinados no âmbito das relações entre saúde, trabalho e ambiente é uma tarefa complexa; porém, é um desafio que precisa ser enfrentado por diversos setores, governamentais ou não, envolvidos com a garantia de uma melhor qualidade de trabalho e vida para a população do país.

\section{Agradecimentos}

Os autores agradecem ao Conselho Nacional de Desenvolvimento Científico e Tecnológico (CNPq) pelo auxílio recebido para a realização do projeto de pesquisa, Processo n ${ }^{\circ} 55193 / 2006-3$, Edital 18/2006. Os autores agradecem também à equipe de pesquisa do projeto "Avaliação do risco à saúde humana decorrente do uso de agrotóxicos (defensivos agrícolas) na agricultura e pecuária na Região Centro-Oeste”, que contou com diversos profissionais e alunos da Universidade Federal do Mato Grosso, da Universidade Federal do Mato Grosso do Sul, da Universidade de Brasília e da Escola Nacional de Saúde Pública Sergio Arouca/Fiocruz.

\section{Contribuições da autoria}

Belo, M. S. S. P.; Peres, P.: elaboraram o desenho do artigo e participaram das etapas de análise dos dados, redação e revisão do material. Também são responsáveis pela elaboração e condução da tese de doutorado da qual este manuscrito é parte integrante. Moreira, J. C.; Pignati, W.: participaram do desenho do projeto maior, ao qual este artigo está integrado, bem como do levantamento e da análise dos dados e da revisão do artigo. Dores, E. F. G. C.: coordenou as análises ambientais realizadas ao longo do projeto e participou do levantamento e da análise dos dados.

\section{Referências}

AGÊNCIA NACIONAL DE VIGILÂNCIA SANITÁRIA. Resolução RDC 48 de 07/07/2008. Brasília: Anvisa, 2008. Disponível em: <http://e-legis.anvisa.gov.br/ leisref/public/showAct.php?id=31728\&word=>. Acesso em: 31 fev. 2012.

Critérios para a classificação toxicológica

de agrotóxicos. Brasília: Anvisa, 2011.

Disponível em: <http://portal.anvisa.gov.br/

wps/wcm/connect/Anvisa+Portal/Anvisa/Inicio/

Agrotoxicos $+\mathrm{e}+$ Toxicologia/Publicacao+Agrotoxico +

Toxicologia/Manual+de+Procedimentos + para + Anali se+Toxicologica $>$. Acesso em: 31 jan. 2012.

ARCURY, T. A. et al. Reducing farmworker residential pesticide exposure: evaluation of a lay health advisor intervention. Health Promotion Practice, v. 10, n. 3, p. 447-455, July 2009.

BECKER, H. Segredos e truques da pesquisa. Rio de Janeiro: Jorge Zahar, 2004.

BERNAL, M. H.; SOLOMON, K. R.; CARRASQUILLA, G. Toxicity of formulated glyphosate (glyphos) and cosmo-flux to larval Colombian frogs 1. Laboratory acute toxicity. Journal of Toxicology Environmental Health Part A, v. 72, n. 15-16, p. 961-965, 2009.

BRAKE, D. G.; EVERSON, D. P. A generational study of glyphosate-tolerant soybeans on mouse fetal, postnatal, pubertal and adult testicular development. Food and Chemical Toxicology, v. 42, n. 1, p. 29-36, Jan. 2004.

BRASIL. Ministério da Agricultura, Pecuária e Abastecimento. Agricultura brasileira em números Anuário 2005. Brasília: MAPA, 2005.

$\overline{2009 / 2} \cdot \frac{\text { Projeções do agronegócio: Brasil }}{2010 / 2020 \text {. Brasília: MAPA, } 2010 .}$

CHALUBINSKI, M.; KOWALSKI, M. L. Endocrine disrupters-potential modulators of the immune system and allergic response. Allergy, v. 61, n. 11, p. 13261335, Nov. 2006.

CURWIN, B. et al. Pesticide use and practices in an Iowa farm family pesticide exposure study. Journal of Agricultural and Safety and Health, v. 8, n. 4, p. 423433, Nov. 2002. 
DALLEGRAVE, E. et al. The teratogenic potential of the herbicide glyphosate-Roundup in Wistar rats. Toxicology Letters, v. 142, n. 1-2, p. 45-52, Apr. 2003.

EJAZ, S. et al. Endocrine disrupting pesticides: a leading cause of cancer among rural people in Pakistan. Experimental Oncology, v. 26, n. 2, p. 98-105, June 2004.

FARIA, N. M.; ROSA, J. A.; FACCHINI, L. A. Poisoning by pesticides among family fruit farmers, Bento Gonçalves, Southern Brazil. Revista de Saúde Pública, São Paulo, v. 43, n. 2, p. 335-344, abr. 2009.

FUNDAÇÃO OSWALDO CRUZ. Avaliação do risco à saúde humana decorrente do uso de agrotóxicos (defensivos agrícolas) na agricultura e pecuária na Região Centro-Oeste. Projeto de Pesquisa - Edital MCT - CNPq/CT-SAÚDE - nº 18/2006. Rio de Janeiro: Fiocruz, 2006.

GASNIER, C. et al. Glyphosate-based herbicides are toxic and endocrine disruptors in human cell lines. Toxicology, v. 262, n. 3 p. 184-191, Aug. 2009.

GIL, F; PIA, A. Biomarkers as biological indicators of xenobiotic exposure. Journal of Applied Toxicology, v. 21, n. 4, p. 245-255, 2001.

HERAS-MENDAZA, F. et al. Erythema multiforme-like eruption due to an irritant contact dermatitis from a glyphosate pesticide. Contact Dermatitis, v. 59, n. 1, p. 54-56, July 2008.

HOKANSON, R. et al. Alteration of estrogen-regulated gene expression in human cells induced by the agricultural and horticultural herbicide glyphosate. Human \& Experimental Toxicology, v. 26, n. 9, p. 747752, Sept. 2007.

\section{INSTITUTO DE DEFESA AGROPECUÁRIA DO} ESTADO DO MATO GROSSO. Banco de dados do sistema de informações sobre agrotóxicos. Instituto de Defesa Agropecuária do Estado do Mato Grosso, Cuiabá/MT. Cuiabá: Indea, 2008. CD-ROM.

KLAASSEN, C. D.; AMDUR, M. O.; DOULL, J. Casarett $\&$ Doull's Toxicology: the basic science of poisons. 3. ed. New York: MacMillan Publishing, 2001.

LONDON, L.; BAILIE, R. Challenges for improving surveillance for pesticide poisoning: policy implications for developing countries. International Journal of Epidemiology, v. 30, n. 3, p. 564-570, June 2001.

MIRANDA, A. C. et al. Neoliberalismo, o uso de agrotóxicos e a crise da soberania alimentar no Brasil. Ciência e Saúde Coletiva, Rio de Janeiro, v. 12, n. 1, p. 7-14, jan./mar. 2007.

MLADINIC, M. et al. Evaluation of genome damage and its relation to oxidative stress induced by glyphosate in human lymphocytes in vitro. Environmental and Molecular Mutagenesis, v. 50, n. 9, p. 800-807, Dec. 2009.
NIELSEN, J. B.; NIELSEN, F; SORENSEN, J. A. Defense against dermal exposures is only skin deep: significantly increased penetration through slightly damaged skin. Archives of Dermatological Research, v. 299, n. 9, p. 423-431, Nov. 2007.

PENAGOS, H. et al. Pesticide patch test series for the assessment of allergic contact dermatitis among banana plantation workers in Panama. Dermatitis, v. 15, n. 3, p. 137-145, Sept. 2004.

PERES, F. Saúde, trabalho e ambiente no meio rural brasileiro. Ciência e Saúde Coletiva, Rio de Janeiro, v. 14, n. 6, p. 1995-2004, 2009.

POLETTA, G. L. et al. Genotoxicity of the herbicide formulation Roundup (glyphosate) in broad-snouted caiman (Caiman latirostris) evidenced by the Comet assay and the Micronucleus test. Mutation Research, v. 672, n. 2, p. 95-102, Jan. 2009.

QUAGHEBEUR, D. et al. Pesticides in rainwater in Flanders, Belgium: results from the monitoring program 1997-2001. Journal of Environmental Monitoring, v. 6, n. 3, p. 182-190, Mar. 2004.

ROUVALIS, A. et al. Determination of pesticides and toxic potency of rainwater samples in western Greece. Ecotoxicology and Environmental Safety, v. 72, n. 3, p. 828-833, Mar. 2009.

SANTOS, L. G. Avaliação da dispersão atmosférica e da deposição úmida de agrotóxicos em Lucas do Rio Verde-MT. 2010. 125 f. Dissertação (Mestrado em Recursos Hídricos)-Universidade Federal do Mato Grosso, Cuiabá, 2010.

SARCINELLI, P. N. Estudo dos níveis de pesticidas organoclorados persistentes em mulheres grávidas e lactantes no Rio de Janeiro. 2001. 90 f. Tese (Doutorado em Biologia Celular e Molecular)-Fundação Oswaldo Cruz, Instituto Oswaldo Cruz, Rio de Janeiro, 2001.

SCHEYER, A. et al. Analysis of trace levels of pesticides in rainwater using SPME and GC-tandem mass spectrometry. Analytical and Bioanalytical Chemistry, v. 384, n. 2, p. 475-487, Jan. 2006.

SCHUMMER, C. et al. Analysis of phenols and nitrophenols in rainwater collected simultaneously on an urban and rural site in east of France. Science of the Total Environment, v. 407, n. 21, p. 5637-5643, Oct. 2009.

SHIPP, E. M. et al. Pesticide safety training among farmworker adolescents from Starr County, Texas. Journal of Agricultural Safety \& Health, v. 13, n. 3, p. 311-321, July 2007.

SINDICATO NACIONAL DAS INDÚSTRIAS DE PRODUTOS PARA A DEFESA AGRÍCOLA. Anuário Estatístico 2008. Brasília: Sindag, 2008.

SLAGER, R. E. et al. Rhinitis associated with pesticide use among private pesticide applicators in the agricultural health study. Journal Toxicology Environmental Health Part A, v. 73, n. 20, p. 13821393, Jan. 2010. 
SOARES, W.; MORO, S.; ALMEIDA, R. M. Rural workers' health and productivity: an economic assessment of pesticide use in Minas Gerais, Brazil. Applied Health Economics and Health Policy, v. 1, n. 3, p. 157-164, 2002.

SOLOMON, K. R.; MARSHALL, E. J.; CARRASQUILLA, G. Human health and environmental risks from the use of glyphosate formulations to control the production of coca in Colombia: overview and conclusions. Journal of Toxicology Environmental Health Part A, v. 72, n. 15-16, p. 914-920, 2009.
VARONA, M. et al. Effects of aerial applications of the herbicide glyphosate and insecticides on human health. Biomedica, v. 29, n. 3, p. 456-475, Sept. 2009.

WATANABE, E. et al. Evaluation of a commercial immunoassay for the detection of chlorfenapyr in agricultural samples by comparison with gas chromatography and mass spectrometric detection. Journal of Chromatography A, v. 1074, n. 1-2, p. 145153, May 2005. 